Check for updates

Cite this: RSC Adv., 2020, 10, 2536

\title{
Synthesis of green-emitting carbon quantum dots with double carbon sources and their application as a fluorescent probe for selective detection of $\mathrm{Cu}^{2+}$ ions
}

\begin{abstract}
Jun $\mathrm{Xu}^{\mathrm{a}}$ Congling Wang, ${ }^{a}$ Huizhi $\mathrm{Li}^{\mathrm{b}}$ and Weilin Zhao (D) *a
Green-emitting carbon quantum dots (G-CQDs) were prepared using tartaric acid and bran by one-pot solvothermal treatment and had photoluminescence quantum yields (PLQY) as high as $46 \%$. The morphology of the G-CQDs is characterized by TEM, which shows the average diameter of G-CQDs is approximately $\sim 4.85 \mathrm{~nm}$. The FT-IR spectra display the presence of $-\mathrm{OH}, \mathrm{C}-\mathrm{N}, \mathrm{N}-\mathrm{H}$ and $-\mathrm{COOH}$ on the surface of the G-CQDs. The emission wavelength of the G-CQDs was $~ 539 \mathrm{~nm}$ in the case of $\sim 450 \mathrm{~nm}$ excitation wavelength, which corresponds to the green fluorescence. Furthermore, the GCQDs were used as a fluorescent probe for detection $\mathrm{Cu}^{2+}$ ions, and demonstrated a linear distribution between $\ln \left(F / F_{0}\right)$ and the $\mathrm{Cu}^{2+}$ ions concentration. Specifically, the $\mathrm{Cu}^{2+}$ ion concentration should fall in the G-CQD concentration range of $0-0.5 \mathrm{mM}$ and the detection limit is $0.0507 \mu \mathrm{M}$. Thus, due to the excellent chemical stability and good luminescence performance, these G-CQDs could be excellent probes widely used in detection fields.
\end{abstract}

Received 22nd October 2019
Accepted 9th January 2020

DOI: $10.1039 / c 9 r a 08654 d$

rsc.li/rsc-advances

quantum dots, CQDs have the advantages of good biocompat-

\section{Introduction}

Over the last few years, a novel type of quantum dot materialcarbon quantum dots (CQDs)- has been discovered and prepared, which further broadens the application range of carbon nanomaterials. In addition, CQDs are a member of the quantum dot material family and are a zero-dimensional semiconductor nanocrystal with a nearly spherical shape and a particle size of less than $10 \mathrm{~nm}$. CQDs are generally composed of very few molecules or atoms and can well disperse in water or organic solvent to form a colloid.

In 2004, Xu et al. ${ }^{1}$ used the arc discharge method to prepare carbon nanotubes. When separated and purified by gel electrophoresis, a carbon nanoparticle with fluorescence was accidentally obtained. Therefore, carbon materials with fluorescent properties began to attract people's attention. Two years later, Sun's research group ${ }^{2}$ obtained fluorescent carbon nanoparticles, which were named "carbon quantum dots (CQDs)". Compared with previous carbon nanomaterials and organic dyes, CQDs have unique luminescent properties and overcome the shortcomings of organic dyes, ${ }^{3}$ such as emission spectrum half-peak width, photobleaching, fluorescence lifetime short and toxic. Also, compared with traditional semiconductor metal

${ }^{a}$ School of Materials Science and Engineering, University of Jinan, Jinan 250022, China.E-mail:zhaowl_ujn@126.com

${ }^{b}$ School of Chemistry and Chemical Engineering, University of Jinan, Jinan 250022, China ibility, low toxicity and easy functionalization in addition to the properties of metal quantum dots. ${ }^{4-7}$ Based on the advantages described above, CQDs are widely used in the fields of fluorescent labeling, ${ }^{8-10}$ analytical detection, ${ }^{11-13}$ and safety ink. ${ }^{14}$ Thus, further research on fluorescent CQDs has important theoretical and practical value.

Nowadays there are multiple methods for preparing CQDs, these methods can be summarized into two categories: topdown and bottom-up methods..$^{15}$ The top-down method mainly includes arc discharge method, ${ }^{16}$ laser ablation method, ${ }^{17}$ and electrochemical method; ${ }^{18,19}$ the bottom-up method contains hydrothermal/solvothermal method, ${ }^{20,21}$ combustion method, ${ }^{22}$ pyrolysis method, ${ }^{23}$ microwave method, ${ }^{24}$ et al. Among them, hydrothermal/solvothermal method is the most commonly used method for preparing CQDs due to its advantages of simplicity and ease of operation.

Wang et al. reported the novel N-CQDs were synthesized by a hydrothermal approach using the carbon source (eggplant sepals). The prepared N-CQDs showed outstanding photoluminescence properties with excellent chemical stability in phosphate buffer solution. ${ }^{25}$ Jiang et al. synthesized the novel Ndoped CQDs by hydrothermal method using isoleucine and citric acid as carbon source. Under optimal conditions, the Ndoped CQDs can be applied to detect $\mathrm{Fe}^{3+}$ and presented a fluorescence quenching response to $\mathrm{Fe}^{3+} .{ }^{26}$ Sarkar et al. obtained CQDs with different sizes via a simple hydrothermal method. The fluorescence properties of these CQDs showed 
that with the excitation wavelength increasing, the emissions wavelength for these samples were red shifted, which indicated the prepared CQDs have dependence on excitation wavelength. $^{27}$

With the deepening of research on CQDs, the application of CQDs has become the focus of research. At present, the researches on the application of CQDs has made some progress, mainly in the fields of optoelectronics, catalysis and sensing, ${ }^{28}$ in which the most common and simple application is the detection of metal ions. He et al. developed a facile synthetic method using cabbage juice to synthetic $\mathrm{N}$-doped carbon dots (NCDs) to detect $\mathrm{Fe}^{3+}$ and the detection limit was $1.4 \mathrm{nM}^{29}$ Wang et al. presented the multicolored emission carbon dots to detect $\mathrm{Fe}^{3+}$ and $\mathrm{Cu}^{2+}$ ions through assigning a different colored emission carbon dot to a different metal ion. ${ }^{30}$ Zhang et al. introduced a hydrothermal approach for obtaining N-doped carbon dots (NCDs) from ammonium citrate and urea. The NCDs enable sensitive and selective determination of $\mathrm{Hg}^{2+}$ in tap water. $^{31}$

In order to improve the application prospects of CQDS, researchers have adopted various methods to modify CQDS, most commonly heteroatom doping (such as N, S, P, etc.). ${ }^{29}$ Bran is the by-product of wheat processing flour and is a kind of production waste and mainly contains $\mathrm{C}, \mathrm{N}$ and $\mathrm{O}$ elements. In this paper, we have used tartaric acid and bran as the double carbon source, and the bran contains $\mathrm{N}$ element, so the bran can also be used as the nitrogen source to prepare $\mathrm{N}$-doped green-emitting carbon quantum dots (G-CQDs). In terms of application, we have used the synthesized G-CQDs to detect $\mathrm{Cu}^{2+}$ ions, and discovered that it has a good selectivity and sensitivity to $\mathrm{Cu}^{2+}$ ions. We expect that our work will be able to recycle wastes and improve the environment, and the G-CQDs will have better applications in detection and probe.

\section{Experimental section}

\subsection{Reagents and chemicals}

Tartaric acid, dimethylformamide (DMF), $\mathrm{Na}_{2} \mathrm{HPO}_{4}$, sodium citrate and the metal salts used in the experiment $(\mathrm{KCl}, \mathrm{NaCl}$, $\mathrm{LiNO}_{3}, \mathrm{MgCl}_{2}, \mathrm{ZnCl}_{2}, \mathrm{CaCl}_{2}, \mathrm{CuSO}_{4}, \mathrm{FeSO}_{4}, \mathrm{Fe}\left(\mathrm{NO}_{3}\right)_{3}$ and $\left.\mathrm{Al}\left(\mathrm{NO}_{3}\right)_{3}\right)$ were bought from Alfa Aesar. The above reagents are analytically pure and can be used directly. Bran is production waste and needs further treatment.

\subsection{Preparation of G-CQDs}

The bran was cleaned, dried, and processed through 200 mesh sieve, then the lower part of the sieve was set aside.

G-CQDs were prepared by one-step solvothermal method using tartaric acid as carbon sources, bran as carbon and nitrogen sources and DMF as organic solvent. The specific experimental steps are as follows: tartaric acid and bran (with a molar ratio of 0.7 ) were added into $20 \mathrm{~mL}$ of DMF and transferred to a Teflon container that was packed into an autoclave. The autoclave was heated at $150{ }^{\circ} \mathrm{C}$ for $8 \mathrm{~h}$ in the oven. After the reaction, the autoclave was naturally cooled to room temperature. The black product was then centrifuged and filtrated with filter membrane $(0.22 \mu \mathrm{m})$ and dialyzed by using the Biotech cellulose ester (CE) membrane (MWCO 500D) to obtain G-CQDs. Finally, the G-CQDs solution was added into the mixed solvents of petroleum ether and ethyl acetate to remove unreacted molecules and then the solid was dried at $65{ }^{\circ} \mathrm{C}$ overnight. The schematic diagram of the experimental process was shown in the Fig. 1.

\subsection{Detection of $\mathrm{Cu}^{2+}$ ions with G-CQDs}

The selectivity test of G-CQDs solution to $\mathrm{Cu}^{2+}$ ions: $\mathrm{KCl}, \mathrm{NaCl}$, $\mathrm{LiNO}_{3}, \mathrm{MgCl}_{2}, \mathrm{ZnCl}_{2}, \mathrm{CaCl}_{2}, \mathrm{CuSO}_{4}, \mathrm{FeSO}_{4}, \mathrm{Fe}\left(\mathrm{NO}_{3}\right)_{3}$ and $\mathrm{Al}\left(\mathrm{NO}_{3}\right)_{3}$ were respectively dissolved in DI-water and configured into a metal ion solution with a concentration of $2 \mathrm{mM}$. In the experiment of ion detection, $2 \mathrm{~mL}$ metal ion solution and $2 \mathrm{~mL}$ G-CQDs solution ( $5 \mathrm{mM}$ ) were selected to be mixed and allowed to stirring for $2 \mathrm{~min}$ at room temperature $\left(\mathrm{pH}=7\right.$ in $\mathrm{Na}_{2} \mathrm{HPO}_{4}$ citrate buffer), and then fluorescence spectrophotometer was used for fluorescence spectrum test.

The sensitivity test of G-CQDs solution to $\mathrm{Cu}^{2+}$ ions: the different concentrations of $\mathrm{Cu}^{2+}$ ions $(\mathrm{pH}=7)$ were added into the G-CQDs solution ( $5 \mathrm{mM}$ ) for stand $2 \mathrm{~min}$ at $25{ }^{\circ} \mathrm{C}$. The fluorescence intensity was measured at the $450 \mathrm{~nm}$ excitation wavelength.

\subsection{Characterization}

In this paper, a series of testing methods are applied to study the chemical and optical performances of G-CQDs.

The high-resolution transmission electron microscopic (HRTEM, JEM-1400) and Nanoparticle size and Zeta potential analyzer (Malvern ZS90) were used to describe the size and morphology of G-CQDs. The specific experimental conditions were as follows: the sample was dropped on a copper mesh of 230 mesh ultra-thin carbon film. The maximum tilt angle of the sample stage was \pm 400 , the acceleration voltage was $200 \mathrm{kV}$, the lattice resolution was $0.2 \mathrm{~nm}$, and the dot resolution was $0.34 \mathrm{~nm}$.

A powder X-ray diffractometer (XRD, Bruker D8 ADVANCE) was used to detect the crystallinity of G-CQDs. The specific
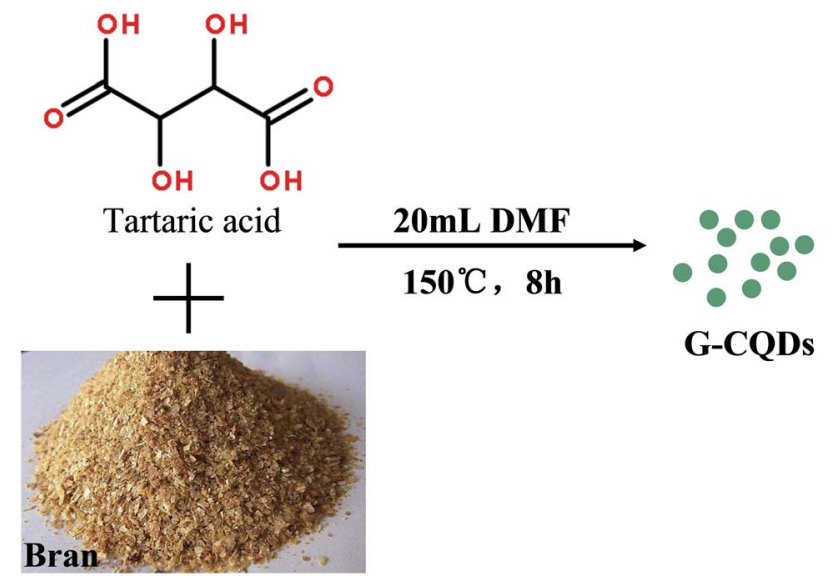

Fig. 1 Schematic illustration of the experimental process of G-CQDs. 


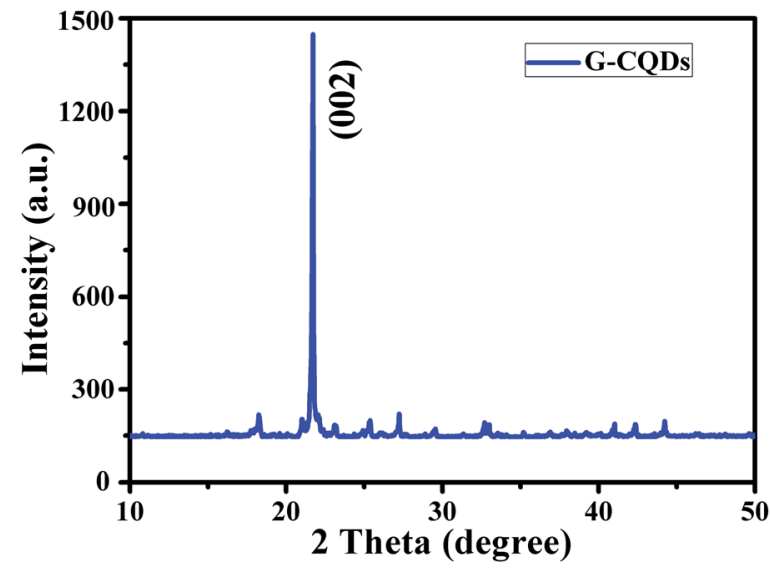

Fig. 2 XRD pattern of the G-CQDs.

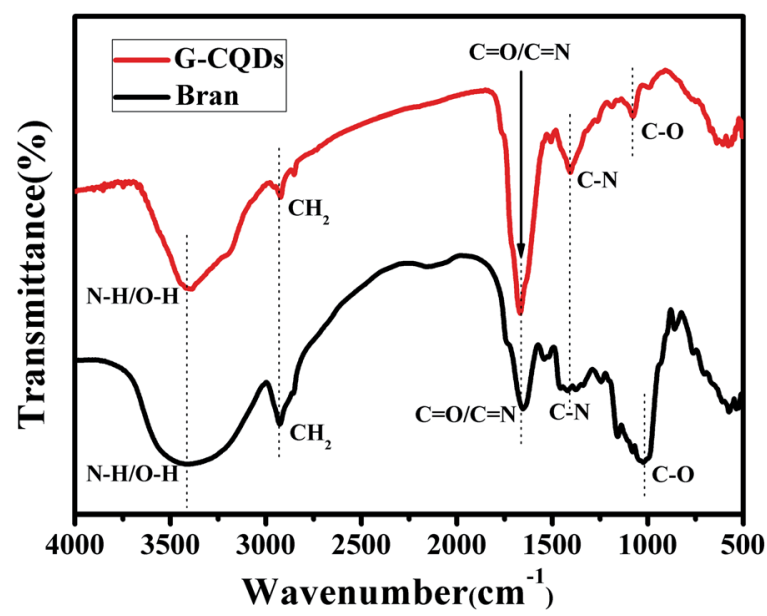

Fig. 3 FT-IR spectra of G-CQDs and bran.

experimental conditions were as follows: the X-ray source used $\mathrm{Cu}$ target $\mathrm{K} \alpha$-ray $(\lambda=0.15406 \mathrm{~nm})$, the tube voltage was $40 \mathrm{kV}$, the tube current was $40 \mathrm{~mA}$, the scanning speed was $5^{\circ} \mathrm{min}^{-1}$, and the scanning range $(2 \theta)$ was $10-80^{\circ}$.

A Thermo NICOLET-380 spectrophotometer was applied to characterize the Fourier transform infrared spectrum (FT-IR) of G-CQDs. The FT-IR had an air scan as the background and the scanning range was $4000-400 \mathrm{~cm}^{-1}$. An X-ray photoelectron spectrometer (Thermo Fisher Scientific, Thermo ESCALAB 250XI) was employed to determine the X-ray photoelectron spectroscopy (XPS) analysis.

A Metash UV-5800 spectrophotometer and Shimadzu RF6500 PC luminescence spectrometer were applied to record the UV-vis absorption spectra and photoluminescence (PL) spectra, respectively. The UV-vis absorption spectra was calibrated using the corresponding solvent as a reference, with a scan interval of $1 \mathrm{~nm}$, a scan range of 300-700 nm, and a scan rate of medium speed. The fluorescence spectrum was measured by placing the sample to be tested in a quartz fourpass cuvette, using a $700 \mathrm{~V}$ neon lamp as the light source, the excitation slit width was $10 \mathrm{~nm}$, the emission slit width was
$10 \mathrm{~nm}$, the scanning interval was $1 \mathrm{~nm}$, and the scanning speed was $500 \mathrm{~nm} \mathrm{~min}{ }^{-1}$, scanning range was $300-700 \mathrm{~nm}$.

\section{Results and discussion}

The result of the XRD analysis of G-CQDs was presented in Fig. 2. The distinct diffraction peak is appeared in the range of $2 \theta=20-24^{\circ}$ corresponding to the plane of (002), this is due to the presence of graphitic carbon. ${ }^{32}$ Furthermore, the lattice spacing of G-CQDs by Bragg formula (1.1) is about $\sim 0.402 \mathrm{~nm}$. This shows that G-CQDs are amorphous structure with $\mathrm{sp}^{2}$ hybrid carbon core of graphitic carbon.

$$
2 d \sin \theta=n \lambda
$$

where $d$ is the lattice spacing of G-CQDs, $\theta$ represents the diffraction angle, $n$ is the reflection series, $\lambda$ represents the wavelength.

To understand the structural characteristics of the prepared G-CQDs, the FT-IR spectrum was employed to analyze the functional groups of G-CQDs. Fig. 3 describes the FT-IR spectrum of the obtained G-CQDs and bran. It can be seen from the FT-IR spectrum (black curve) of the bran that the bran contains $\mathrm{C}-\mathrm{H}_{2}, \mathrm{C}-\mathrm{O}, \mathrm{C}-\mathrm{N}, \mathrm{C}=\mathrm{N}$ and $\mathrm{N}-\mathrm{H}$ bonds, which can be used as a carbon source to prepare the G-CQDs, and as a nitrogen source to provide $\mathrm{N}$ elements for the G-CQDs. From the FT-IR spectrum (red curve) of the G-CQDs, the absorption peak at $3392 \mathrm{~cm}^{-1}$ corresponds to the stretching vibration of the $\mathrm{N}-\mathrm{H}$ and $\mathrm{O}-\mathrm{H}$, indicating that the surface of G-CQDs contains $-\mathrm{NH}_{2}$ and hydrophilic - $\mathrm{OH}$. Meanwhile, the stretching vibration peak for $\mathrm{CH}_{2}\left(2852 \mathrm{~cm}^{-1}\right), \mathrm{C}=\mathrm{O}$ and $\mathrm{C}=\mathrm{N}\left(1668 \mathrm{~cm}^{-1}\right), \mathrm{C}-\mathrm{N}$ $\left(1405 \mathrm{~cm}^{-1}\right)$ and $\mathrm{C}-\mathrm{O}\left(1078 \mathrm{~cm}^{-1}\right)$ illustrates that the surface of G-CQDs is connected to functional groups containing $\mathrm{O}-$ and $\mathrm{N}-$.

The full survey of XPS of G-CQDs was performed to characterize the elemental composition and valence states of G-CQDs and control experiment. The result was shown in Fig. 4. Fig. 4(a) displays the synthesized G-CQDs and the compare experimental CQDs mainly all contain $\mathrm{C}, \mathrm{N}$, and $\mathrm{O}$ elements. This indicates that bran can provide $\mathrm{N}$ element for the preparation of G-CQDs. The XPS full survey spectra of G-CQDs shows an clear peak of $\mathrm{C}$ $1 \mathrm{~s}$ at $\sim 298.9 \mathrm{eV}, \mathrm{O} 1 \mathrm{~s}$ at $\sim 531.4 \mathrm{eV}$ and N $1 \mathrm{~s}$ at $\sim 401.3 \mathrm{eV}$, which is similar with the characteristic peaks found in the N-CQDS prepared using biomass tar and ethylene-diamine solution. ${ }^{33}$ Fig. 4(b) reveals three peaks of the C 1s XPS spectrum, which can be fitted into $\mathrm{sp}^{2}$ and $\mathrm{sp}^{3}$ hybridized carbon atoms in $\mathrm{C}-\mathrm{C} /$ $\mathrm{C}=\mathrm{C}(\sim 285 \mathrm{eV}), \mathrm{C}-\mathrm{N} / \mathrm{C}-\mathrm{O}(\sim 287.1 \mathrm{eV})$ and a carboxyl group $(-\mathrm{COOH})$ at $\sim 288.7 \mathrm{eV}$, separately. Fig. 4(c) shows the N 1s XPS spectrum of G-CQDs, which presents the peak appearing at $\sim 399.8 \mathrm{eV}$ corresponds to a pyridine N. The O 1s XPS spectrum (Fig. 4(d)) of G-CQDs displays that the characteristic peaks at $\sim 531.1 \mathrm{eV}$ and $\sim 532.5 \mathrm{eV}$, corresponding to the $\mathrm{C}=\mathrm{O}$ bond and the $\mathrm{C}-\mathrm{O}$ bond, respectively.

From Fig. 5, the TEM images of G-CQDs were investigated. We can see the particle size of G-CQDs is mainly concentrated in the range of 4-6 $\mathrm{nm}$ and the average particle size is $\sim 4.85 \mathrm{~nm}$ (Fig. 5(a)). From the HR-TEM of G-CQDs (Fig. 5(b)), it can be 

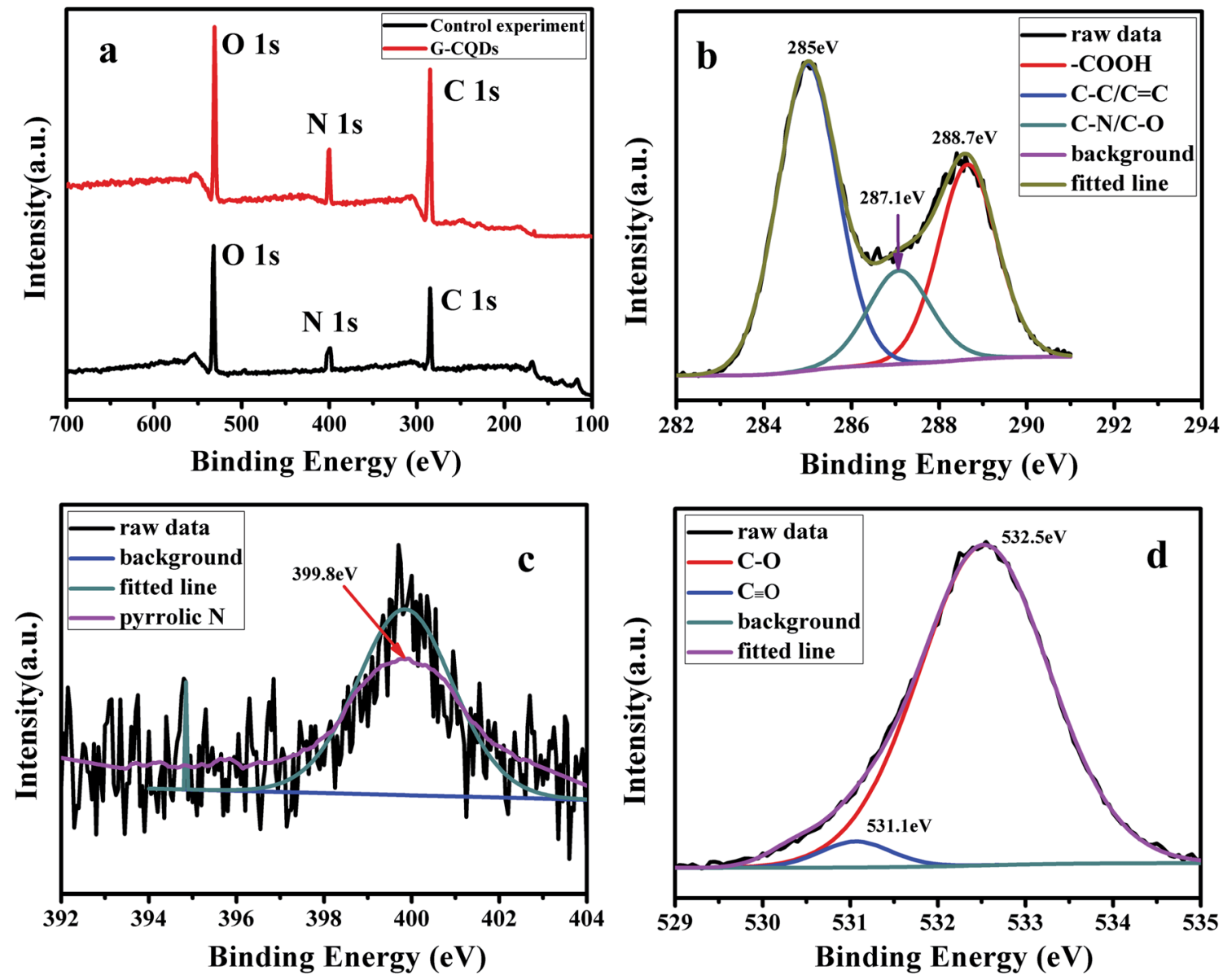

Fig. 4 (a) Full-survey XPS spectrum of G-CQDs and control experiment, (b) C 1s, (c) N 1s and (d) O 1s XPS spectra.

seen the G-CQDs have distinct stripes and the stripe pitch is $0.24 \mathrm{~nm}$.

Fig. 6 displays the photoluminescence (PL) performance of G-CQDs. From Fig. 6(a), the UV absorption spectrum of G-CQDs has a distinct absorption peak at $\sim 350 \mathrm{~nm}$, which may be caused by the $\mathrm{n}-\pi^{*}$ transition of $\mathrm{C}=\mathrm{O} \cdot{ }^{34}$ Meanwhile, it can be seen from the excitation and emission spectrum curves of GCQDs that at $450 \mathrm{~nm}$ excitation wavelength, the optimum fluorescence emission peak is $\sim 539 \mathrm{~nm}$, which showed the green emission and was consistent with the photograph of the inline image. To further explore the PL performance of G-CQDs, the excitation wavelength dependence of G-CQDs was analyzed.
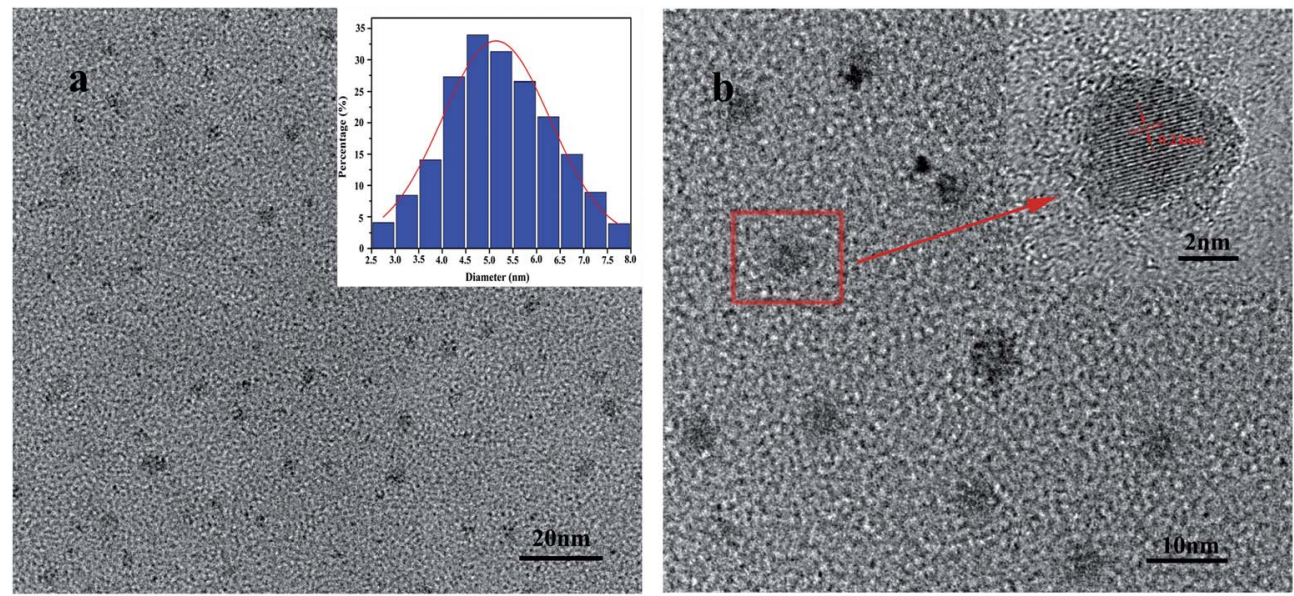

Fig. 5 Morphologies and structural characterization of the G-CQDs: (a) TEM and particle size distribution, (b) HR-TEM. 

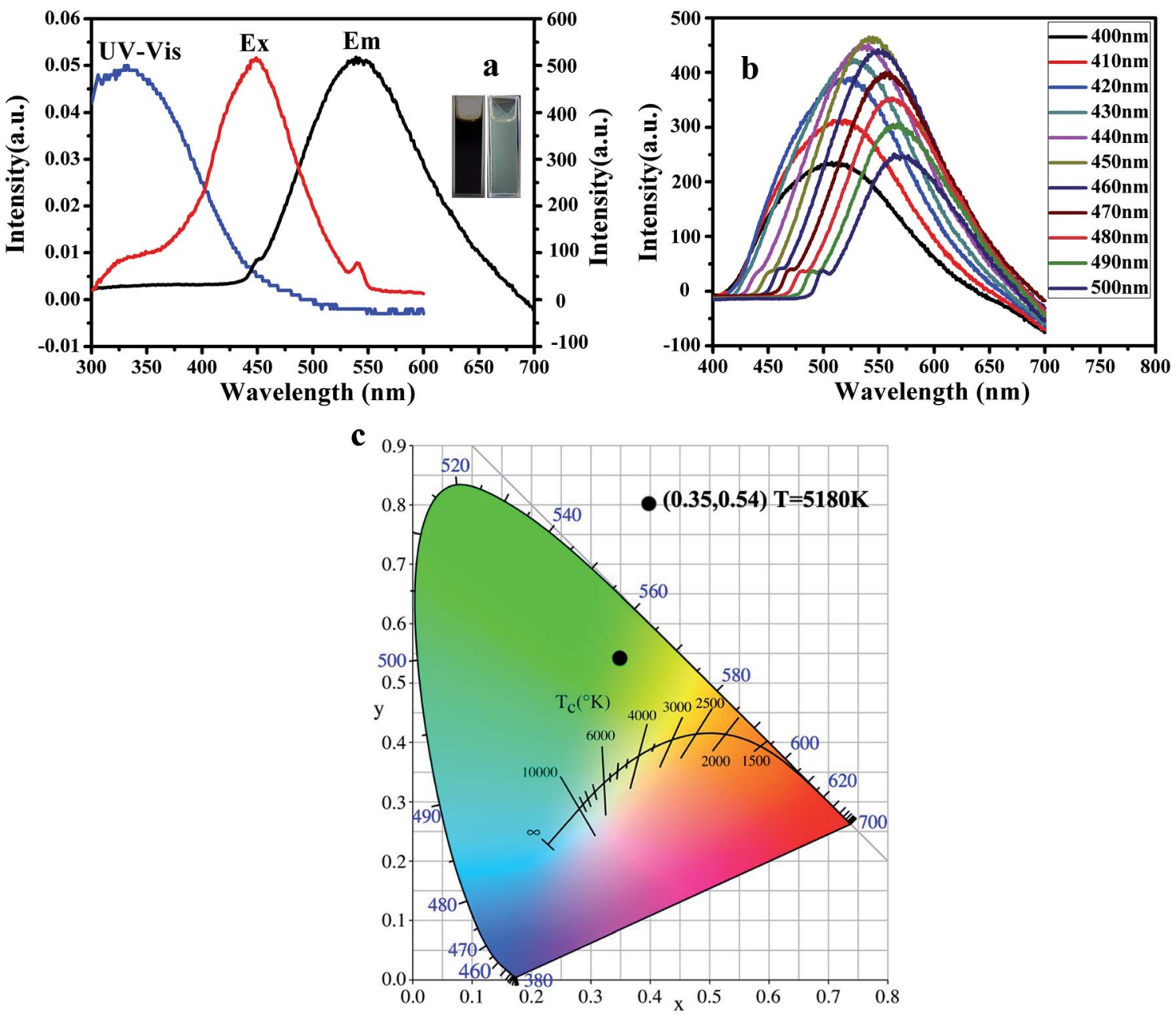

Fig. 6 (a) UV-vis and PLE excitation spectra of G-CQDs (inset: B-CQDs: photographs taken under sunlight and UV light), (b) fluorescence emission spectra of G-CQDs at different excitation wavelengths, (c) color coordinates and color temperature of G-CQDs.

Fig. 6(b) exhibits the fluorescence emission spectra of G-CQDs under different excitation wavelengths. It can be observed that with the increase of excitation wavelength, the fluorescence intensity of the G-CQDs first increases and then decreases; and

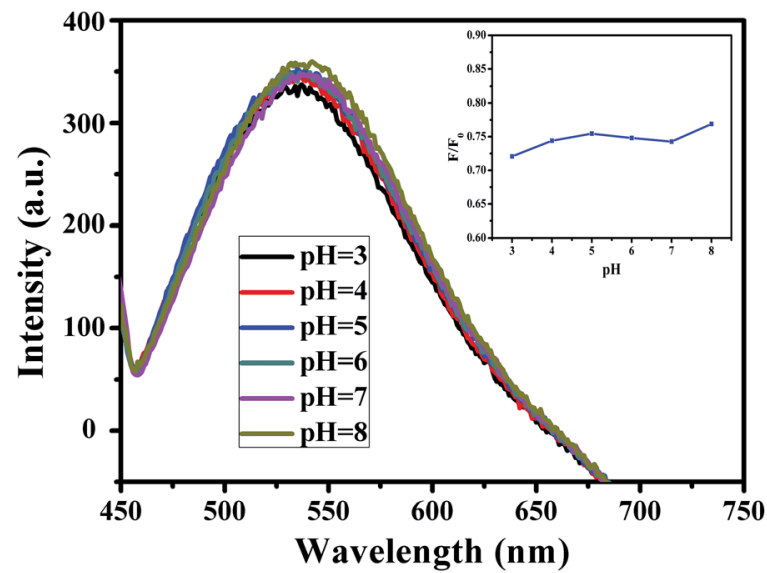

the fluorescence intensity of G-CQDs is the strongest at the $\sim 450 \mathrm{~nm}$ excitation wavelength. Meanwhile, from Fig. 6(c), under the excitation of $\sim 450 \mathrm{~nm}$, the color coordinates of GCQDs are in the green region $(\sim 0.35, \sim 0.54)$. The color temperature is $\sim 5180 \mathrm{~K}$, which calculated by eqn (1.2) and (1.3). In addition, according to the formula (1.4), the calculated photoluminescence quantum yields (PL QY) of the G-CQDs by relative method can reach up to $46 \%$.

$$
\begin{gathered}
T=-437 n^{3}+3601 n^{2}-6861 n+5514.31 \\
n=(x-0.332) /(y-0.1858) \\
\phi_{\text {sample }}=\frac{A_{\text {standard }} \phi_{\text {standard }} \int F_{\text {sample }}}{A_{\text {sample }} \int F_{\text {standard }}}
\end{gathered}
$$

where $A_{\text {standard }}$ and $A_{\text {sample }}$ are the absorbance of the standard (quinine sulfate) and G-CQDs under excitation wavelength, $\int F_{\text {standard }}$ and $\int F_{\text {sample }}$ express the integral area of the emission spectrum of the standard (quinine sulfate) and G-CQDs under the same condition.

Fig. 7 The effect of $\mathrm{pH}$ on the fluorescence intensity of G-CQDs. 


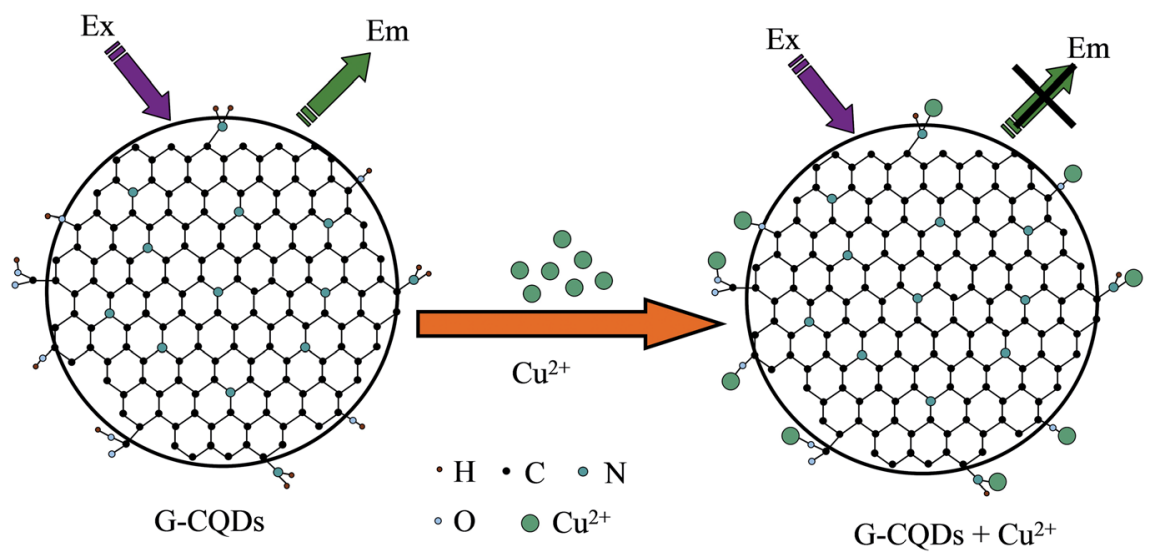

Fig. 8 Fluorescence quenching mechanism of G-CQDs in the presence of $\mathrm{Cu}^{2+}$ ions.

Moreover, the effect of $\mathrm{pH}$ on the fluorescence intensity of GCQDs was investigated (Fig. 7). As the $\mathrm{pH}$ increased from 3 to 8, the fluorescence intensity of G-CQDs remained basically unchanged, which means in the acid and alkali solution, the fluorescence intensity of G-CQDs only changed slightly. This might due to the surface of G-CQDs contains functional groups such as $-\mathrm{OH},-\mathrm{NH}$, and the stability of the functional group is changed under different $\mathrm{pH}$ conditions, so that these functional groups are protonated or deprotonated, but only the charge transfer occurs on the surface of the G-CQDs, and the core structure is still stable. The above shows that G-CQDs are not pH dependent. ${ }^{35}$

In this paper, based on the excellent PL performance and chemical stability of G-CQDs, we studied the application of GCQDs in metal ion detection. So far, researchers have done a series of studies on the application of CQDs to detect $\mathrm{Cu}^{2+}$ ions. Liang et al. reported a kind of multicolor fluorescent $\mathrm{N}$ CDots obtained by hydrothermal treatment, which can be used as a fluorescent probe for the detection of $\mathrm{Fe}^{3+}$ ions. And they found the N-CDots showed lower detection limit (7.6 nM) and higher selectivity during the concentration of $\mathrm{Fe}^{3+}$ ions in tap water. ${ }^{36} \mathrm{Wu}$ et al. synthesized CQDs from vitamin B1 via a hydrothermal process and the B1-CQDs could be utilized as a reliable probe to detect of $\mathrm{Fe}^{3+}$ ions with the detection limit as low as $177 \mathrm{nM} .^{37}$ Murugan et al. indicated the quenching efficiency and the concentration of $\mathrm{Cu}^{2+}$ ions possessed a linear distribution with the concentration range of $0-100 \mu \mathrm{M}\left(R^{2}=\right.$ 0.9918). ${ }^{38}$ The study of Moonrinta et al. presented that CDs were compound using edible yogurt via a solvothermal process and the CDs was employed as fluorescent probe to detect formic acid vapor and metal ions. The formic acid concentration was detected by adjusting the light transmission of CDs and the detection limit was $7.3 \% \mathrm{v} / \mathrm{v}^{39}$

$\mathrm{Cu}^{2+}$ ions are kind of heavy metal ion. When a large amount of $\mathrm{Cu}^{2+}$ ions remain in the human body, it is easy to burden the organs in the body. Therefore, it is of vital important to select a simple and easy method to detect $\mathrm{Cu}^{2+}$ ions. The mechanism of CQDs and detection of $\mathrm{Cu}^{2+}$ ions is due to the coordination or complexation of functional groups such as hydroxyl groups $(-\mathrm{OH})$ and carboxyl groups $(-\mathrm{COOH})$ on the surface with $\mathrm{Cu}^{2+}$ ions, so that the fluorescence quenching of CQDs can realize the selectivity and sensitivity detection of $\mathrm{Cu}^{2+}$ ions. The reaction principle of G-CQDs reacting with $\mathrm{Cu}^{2+}$ is similar with that reported by Van, ${ }^{\mathbf{4 0}}$ and the reaction mechanism diagram is shown in Fig. 8.
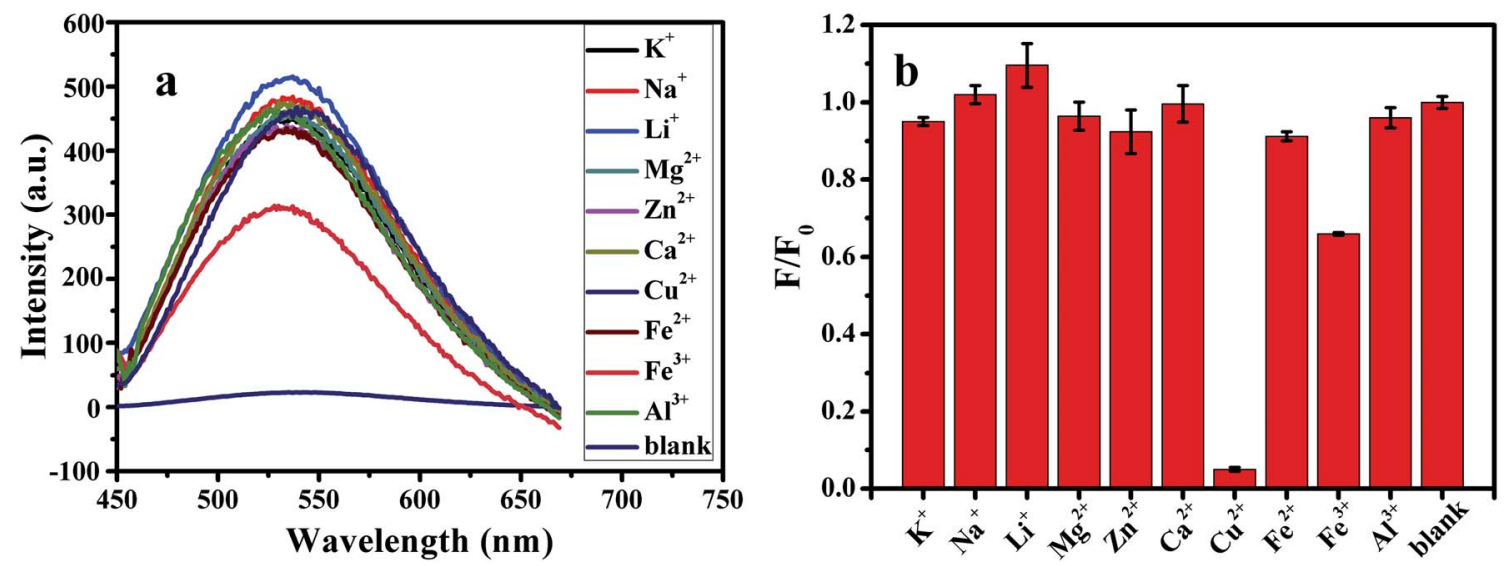

Fig. 9 Effect of different metal ions on G-CQDs: (a) fluorescence intensity, (b) fluorescence quenching rate. 

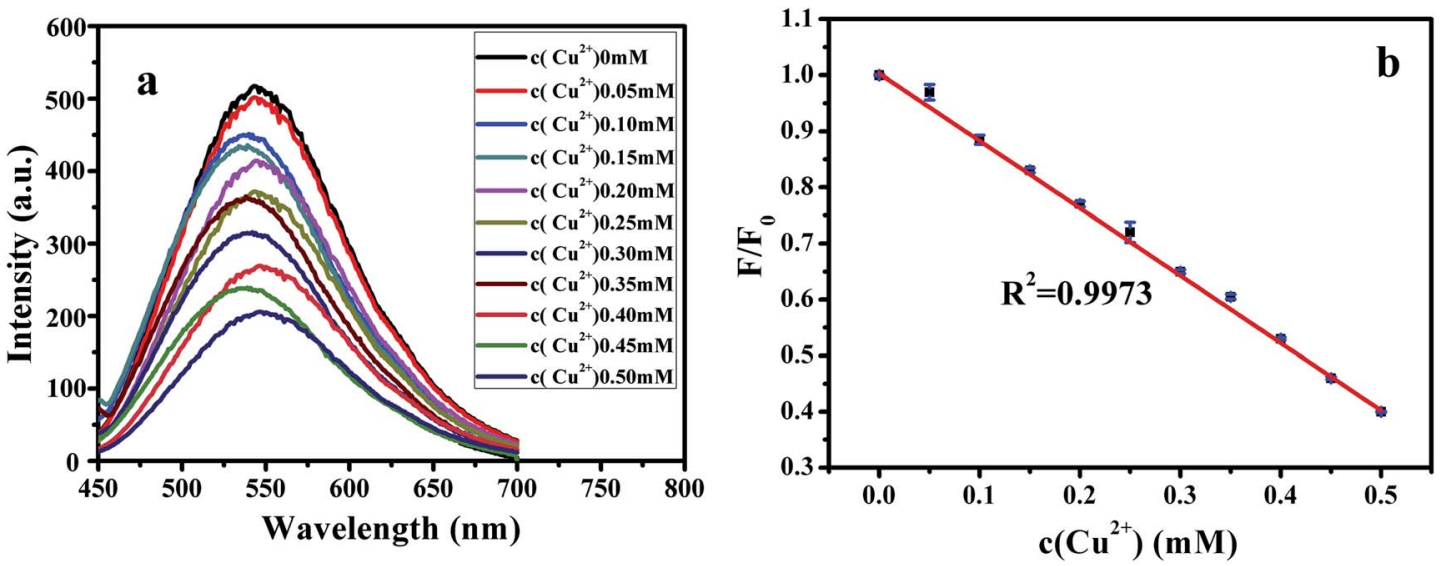

Fig. 10 Effect of $\mathrm{Cu}^{2+}$ ions concentration on the fluorescence of G-CQDs: (a) fluorescence intensity, (b) linear relation graph.

In this paper, the effects of different metal ions $\left(\mathrm{K}^{+}, \mathrm{Na}^{+}, \mathrm{Li}^{+}\right.$, $\left.\mathrm{Mg}^{2+}, \mathrm{Zn}^{2+}, \mathrm{Ca}^{2+}, \mathrm{Cu}^{2+}, \mathrm{Fe}^{2+}, \mathrm{Fe}^{3+}, \mathrm{Al}^{3+}\right)$ on G-CQDs were investigated firstly (Fig. 9(a)). The concentration of each metal ion is $2 \mathrm{mM}$. $\mathrm{Cu}^{2+}$ ions have the highest degree of fluorescence quenching for G-CQDs, and the fluorescence quenching rate is as high as $94 \%$ (Fig. 9(b)). Other metal ions have almost no interference, indicating that G-CQDs as a fluorescent probe have good selectivity to $\mathrm{Cu}^{2+}$ ions.

Fig. 10(a) shows the spectrum of fluorescence quenching of different concentrations of $\mathrm{Cu}^{2+}$ ions and G-CQDs. Fig. 10(b) indicates a linear distribution between the concentration of $\mathrm{Cu}^{2+}$ ions and the fluorescence quenching rate of G-CQDs. The $\mathrm{Cu}^{2+}$ ions concentration in the range of $0-0.5 \mathrm{mM}$ has a linear distribution with the quenching rate $F / F_{0}$, where $F_{0}$ is and $F$ is the fluorescence intensities of after the addition $\mathrm{Cu}^{2+}$ ions. $F / F_{0}$ $=-1.213\left[\mathrm{Cu}^{2+}\right]+1.0143$, linear correlation coefficient $\left(R^{2}\right)$ is 0.9973 , the detection limit is $0.0507 \mu \mathrm{M}$ (according to the formula of $3 S_{0} / k$, where $S_{0}$ is the standard deviation of the blank sample ( $n=11)$, and $k$ is the slope of the linear equation).

Table 1 shows the comparison of the detection limit of the GCQDs to $\mathrm{Cu}^{2+}$ ions with other detection methods. ${ }^{41-45}$ We can see that the detection limit of G-CQDs for $\mathrm{Cu}^{2+}$ ions is relatively low, indicating that the prepared G-CQDs has excellent sensitivity to $\mathrm{Cu}^{2+}$ ions.

To explore the effects of different concentrations of $\mathrm{Cu}^{2+}$ ions on the fluorescence lifetime of G-CQDs, we studied the fluorescence lifetime of G-CQDs under different concentrations of $\mathrm{Cu}^{2+}$ ions (Fig. 11). From Fig. 11, it can be seen that the G-CQDs' fluorescence life was $6.34 \mathrm{~ns}$. The fluorescence life of G-CQDs decreased gradually with the increase of $\mathrm{Cu}^{2+}$ ions concentration, and the fluorescence life is $6.32 \mathrm{~ns}, 6.31 \mathrm{~ns}, 6.30 \mathrm{~ns}, 5.89 \mathrm{~ns}$ and $5.56 \mathrm{~ns}$ (the concentration $\mathrm{Cu}^{2+}$ ions is $0,1,2,3,4,5 \mathrm{mM}$ ), respectively. The results of fluorescence life displays that the fluorescence quenching of the G-CQDs caused by $\mathrm{Cu}^{2+}$ ions may be due to the electron transfer from the excited state of the GCQDs to $\mathrm{Cu}^{2+}$ ions.

To further illustrate the synthesized fluorescent G-CQDs can be used for detection $\mathrm{Cu}^{2+}$ ions, the standard recovery tests were

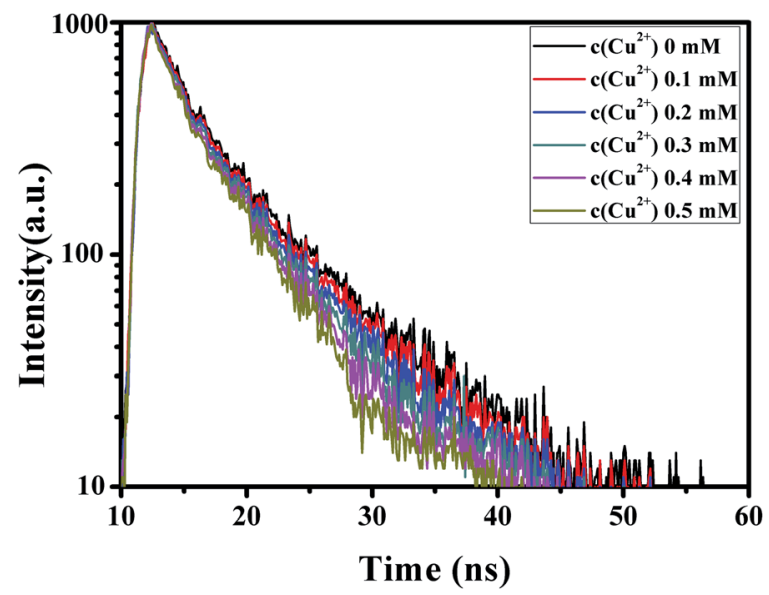

Fig. 11 The effects of different concentrations of $\mathrm{Cu}^{2+}$ ions on the fluorescence lifetime of G-CQDs.

Table 1 Comparison of the detection limit of the G-CQDs to $\mathrm{Cu}^{2+}$ with other detection methods

\begin{tabular}{lll}
\hline Materials & Type of CQDs & Detection limit \\
\hline O-Phenylendiamine & N-CDs & $0.28 \mu \mathrm{M}$ \\
Citric acid and polyethylenimine (PEI) & N-CDs & 41 \\
Sulfuric acid and [C4mim][Cys] carbonization & N/S-CDs & $0.090 \mu \mathrm{M}$ \\
CdTe and thioglycolic acid (TGA) & CD@SiO $@$ CdTe & $0.18 \mu \mathrm{M}$ \\
Citric acid, ethylenediamine and 1,4,8,11-tetraazacyclo tetradecane & Cyclam-functionalized carbon dots & $0.096 \mu \mathrm{M}$ \\
Tartaric acid and bran & N-CQDs & $0.13 \mu \mathrm{M}$ \\
\end{tabular}


Table 2 Determination results of $\mathrm{Cu}^{2+}$ ions in water samples

\begin{tabular}{lllll}
\hline Sample & $\begin{array}{l}\mathrm{Cu}^{2+} \text { added } \\
(\mu \mathrm{M})\end{array}$ & $\begin{array}{l}\mathrm{Cu}^{2+} \text { found } \\
(\mu \mathrm{M})\end{array}$ & $\begin{array}{l}\mathrm{RSD}(n \\
=3, \%)\end{array}$ & Recovery $(\%)$ \\
\hline Mineral water & 300 & 323.16 & 0.10 & 107.72 \\
Tap water & 300 & 335.41 & 0.19 & 111.80 \\
DI-water & 300 & 327.34 & 0.12 & 109.11
\end{tabular}

conducted on mineral water, tap water and DI-water. The detect results are shown in Table 2. As we can see from Table 2, no matter in mineral water, tap water or DI-water, the synthesized fluorescent G-CQDs have a good recovery rate for detection (ranged from $107.72 \%$ to $111.80 \%$ ) and a small relative standard deviation (ranged from $0.10 \%$ to $0.19 \%$ ), indicating that this method has a high accuracy.

\section{Conclusion}

In summary, the N-doped G-CQDs $(\sim 4.85 \mathrm{~nm})$ were prepared through a simple solvothermal treatment from tartaric acid and bran. Based on this, the fluorescence properties and applications of the prepared G-CQDs were analyzed. The G-CQDs present green emission with the maximum peak centered at $\sim 539 \mathrm{~nm}$ at the $\sim 450 \mathrm{~nm}$ optimum excitation wavelength and the PL QY of the G-CQDs reach up to $46 \%$. Under excitation of $\sim 450 \mathrm{~nm}$, the color coordinates and color temperature of GCQDs were calculated to be $(\sim 0.35, \sim 0.54)$ and $\sim 5180 \mathrm{~K}$, respectively. The results indicated the color coordinates were consistent with the maximum emission wavelength of the GCQDs. Furthermore, the G-CQDs were employed as fluorescent probe to detect $\mathrm{Cu}^{2+}$ ions with high selectivity and sensitivity, and the detection limit is $0.0507 \mu \mathrm{M}$. Meanwhile, we verified that the G-CQDs have high accuracy in the detection of $\mathrm{Cu}^{2+}$ ions in actual water samples. Thus, we expected that the GCQDs synthesized in this paper will be widely applied in the detection fields.

\section{Conflicts of interest}

The authors declare that they have no known competing financial interests or personal relationships that could have appeared to influence the work reported in this paper.

The authors declare the following financial interests/ personal relationships which may be considered as potential competing interests: we declare that we have no financial and personal relationships with other people or organizations that can inappropriately influence our work, there is no professional or other personal interest of any nature or kind in any product, service and/or company that could be construed as influencing the position presented in, or the review of, the manuscript entitled, "Synthesis of green-emitting carbon quantum dots with double carbon sources and their application as a fluorescent probe for selective detection of $\mathrm{Cu}^{2+}$ ions".

\section{Acknowledgements}

The work was supported in part by the National Natural Science Foundation of China (No. 51402125), Shandong key research and development plan (No. 2017GGX20127).

\section{References}

1 X. Xu, R. Ray, Y. Gu, H. J. Ploehn, L. Gearheart, K. Raker and W. A. Scrivens, J. Am. Chem. Soc., 2004, 126, 12736-12737.

2 Y. P. Sun, B. Zhou, Y. Lin, K. A. S. Fernando, P. Pathak, M. J. Meziani, B. A. Harruff, X. Wang, H. Wang, P. G. Luo, H. Yang, M. E. Kose, B. Chen and L. M. Veca, J. Am. Chem. Soc., 2006, 128, 7756-7757.

3 H. Mattoussi, J. M. Mauro, E. R. Golaman and G. P. Anderson, J. Am. Chem. Soc., 2000, 122, 12142-12150.

4 X. Guo, Y. Zhu, L. Zhou, L. Zhang, Y. You, H. Zhang and J. Hao, RSC Adv., 2018, 8, 38091-38099.

5 M. Chen, W. Wu, Y. Chen, Q. Pan, Y. Chen, Z. Zheng, Y. Zheng, L. Huang and S. Weng, RSC Adv., 2018, 8, 4143241438.

6 B. Wu, X. Liu, X. Shi, W. Han, C. Wang and L. Jiang, RSC Adv., 2019, 9, 8340-8349.

7 S. Song, K. Wu, H. Wu, J. Guo and L. Zhang, RSC Adv., 2019, 9, 7362-7374.

8 M. Geszke-Moritz and M. Moritz, Mater. Sci. Eng. C, 2013, 33, 1008-1021.

9 C. Guan, Y. Zhao, Y. Hou, G. Shan, D. Yan and Y. Liu, Talanta, 2018, 182, 314-323.

10 Y. Feng, D. Zhong, H. Miao and X. Yang, Talanta, 2015, 140, 128-133.

11 M. Amjadi, R. Shokri and T. Hallaj, Spectrochim. Acta, Part A, 2015, 153, 619-624.

12 L. Rao, Y. Tang, Z. Li, X. Ding, G. Liang, H. Lu, C. Yan, K. Tang and B. Yu, Mater. Sci. Eng. C, 2017, 81, 213-223.

13 Q. Song, Y. Ma, X. Wang, T. Tang, Y. Song, Y. Ma, G. Xu, F. Wei, Y. Cen and Q. Hu, J. Colloid Interface Sci., 2018, 516, 522-528.

14 Z. Song, T. Lin, L. Lin, S. Lin, F. Fu, X. Wang and L. Guo, Angew. Chem., Int. Ed., 2016, 128, 2823-2827.

15 S. N. Baker and G. A. Baker, Angew. Chem., Int. Ed., 2010, 49, 6726-6744.

16 M. Bottini, C. Balasubramanian, M. I. Dawson, A. Bergamaschi, S. Bellucci and T. Mustelin, J. Phys. Chem. $B, 2005,110,831-836$.

17 R. L. Calabro, D. S. Yang and D. Y. Kim, J. Colloid Interface Sci., 2018, 527, 132-140.

18 S. Ahirwar, S. Mallick and D. Bahadur, ACS Omega, 2017, 2, 8343-8353.

19 H. Huang, S. Yang, Q. Li, Y. Yang, G. Wang, X. You, B. Mao, H. Wang, Y. Ma, P. He, Z. Liu, G. Ding and X. Xie, Langmuir, 2018, 34, 250-258.

20 J. D. Xie, G. W. Lai and M. M. Huq, Diamond Relat. Mater., 2017, 79, 112-118.

21 T. Shen, Q. Wang, Z. Guo, J. Kuang and W. Cao, Ceram. Int., 2018, 44, 11828-11834. 
22 S. Zhang, L. Zhang, L. Huang, G. Zheng, P. Zhang, Y. Jin, Z. Jiao and X. Sun, J. Lumin., 2019, 206, 608-612.

23 Q. L. Chen, C. F. Wang and S. Chen, J. Mater. Sci., 2013, 48, 2352-2357.

24 H. Li, F. Q. Shao, H. Huang, J. J. Feng and A. J. Wang, Sens. Actuators, B, 2016, 226, 506-511.

25 Y. Wang and X. Jiang, Sci. China: Chem., 2016, 59, 836-842.

26 Y. Jiang, Q. Han, C. Jin, J. Zhang and B. Wang, Mater. Lett., 2015, 141, 366-368.

27 S. Sarkar, D. Banerjee, U. K. Ghorai, N. S. Das and K. K. Chattopadhyay, J. Lumin., 2016, 178, 314-323.

28 Q. Zhong, Y. Chen, A. Su and Y. Wang, Sens. Actuators, B, 2018, 273, 1098-1102.

29 Y. He, L. Liang, Q. Liu, J. Guo, D. Liang and H. Liu, RSC Adv., 2017, 7, 56087-56092.

30 Y. Wang, Q. Chang and S. Hu, Sens. Actuators, B, 2017, 253, 928-933.

31 Y. Zhang, N. Jing, J. Zhang and Y. Wang, Int. J. Environ. Anal. Chem., 2017, 97, 841-853.

32 H. Zhu, X. Wang, X. Y. Li, Z. Wang, Z. F. Yang and X. Yang, Chem. Commun., 2009, 5118-5120.

33 X. Deng, Y. Feng, H. Li, Z. Du, Q. Teng and H. Wang, Particuology, 2018, 41, 94-100.

34 H. Nie, M. Li, Q. Li, S. Liang, Y. Tan, L. Sheng, W. Shi and X. A. Zhang, Chem. Mater., 2014, 26, 3104-3112.
35 X. Li, H. Wang, Y. Shimizu, A. Pyatenko, K. Kawaquchi and N. Koshizaki, Chem. Commun., 2011, 47, 932-934.

36 Y. Liang, Y. Liu, S. Li, B. Lu, C. Liu, H. Yang, X. Ren and Y. Hou, Opt. Mater., 2019, 89, 92-99.

37 F. Wu, M. Yang, H. Zhang, S. Zhu, X. Zhu and K. Wang, Opt. Mater., 2018, 77, 258-263.

38 N. Murugan, M. Prakash, M. Jayakumar, A. Sundaramurthy and A. K. Sundramoorthy, Appl. Surf. Sci., 2019, 476, 468480.

39 S. Moonrinta, B. Kwon, I. In, S. Kladsomboon, W. Sajomsang and P. Paoprasert, Opt. Mater., 2018, 81, 93-101.

40 T. T. Van and C. W. Mook, Curr. Appl. Phys., 2018, 18, 12551260.

41 W. Lv, M. Lin, R. Li, Q. Zhang, H. Liu, J. Wang and C. Huang, Chin. Chem. Lett., 2019, 7, 1410-1414.

42 A. S. Castillo, M. A. Avidad, C. Pritz, M. C. Robles, B. Fernández, M. J. R. Rama, A. M. Fernández, A. L. Fernández, F. S. Gonzalez, A. S. Fischer and L. F. C. Vallvey, Chem. Commun., 2013, 49, 1103.

43 P. P. Xu, C. F. Wang, D. Sun, Y. J. Chen and K. L. Zhuo, Chem. Res. Chin. Univ., 2015, 31, 730.

44 H. B. Rao, W. Liu, Z. W. Lu, Y. Y. Wang, H. W. Ge, P. Zou, X. X. Wang, H. He, X. Y. Zeng and Y. J. Wang, Microchim. Acta, 2016, 183, 581.

45 J. Chen, Y. Li, K. Lv, W. B. Zhong, H. Wang, Z. Wu, P. G. Yi and J. H. Jiang, Sens. Actuators, B, 2016, 224, 298. 